\title{
Role of Flute Modes in the GAMMA10 Tandem Mirror
}

\author{
Isao KATANUMA, Kotaro YAGI, Nobuyuki ICHIOKA, Shun MASAKI, Yosuke NAKASHIMA, \\ Makoto ICHIMURA and Tsuyoshi IMAI \\ Plasma Research Center, University of Tsukuba, 1-1-1 Tennoudai, Tsukuba, Ibaraki 305-8577, Japan
}

(Received 5 December 2009 / Accepted 16 March 2010)

\begin{abstract}
The steady state in the GAMMA10 tandem mirror, in which radial transport due to flute mode turbulence is balanced by plasma heating and production, is investigated. The basic equations used here exclude the Alfvén and acoustic modes, which are unnecessary for transport due to the flute modes. The basic equations can be applied to the magnetic divertor if the system is assumed to be axisymmetric. High-pressure effects in non-axisymmetric magnetic fields in GAMMA10 are taken into the magnetic specific volume. Numerical calculation is performed to investigate the transport process due to flute mode fluctuations. Mild plasma heating with production leads the system to a steady state, whereas strong heating with production leads the system into sawtooth-like oscillation due to strong flute mode turbulence.
\end{abstract}

(C) 2010 The Japan Society of Plasma Science and Nuclear Fusion Research

Keywords: flute mode, interchange mode, mirror, MHD, simulation

DOI: $10.1585 /$ pfr.5.S2043

\section{Introduction}

The GAMMA10 tandem mirror stabilizes the flute modes by creating high pressure in the non-axisymmetric anchor mirror cells rather than by line-tying effects [1], as shown in Fig. 1. The plasma pressures in the anchor mirror cells and in the central cell are created independently by different heating systems.

Figure 2 shows the time evolutions of the plasma line density $\left(n \ell_{\mathrm{cc}}\right)[\mathrm{Fig} .2(\mathrm{~b})]$ and diamagnetism $\left(D M_{\mathrm{cc}}\right)$ [Fig. 2(c)] measured in the central cell [2]. When electron cyclotron resonance heating at the plug region (plug ECRH) was turned on, $n \ell_{\mathrm{cc}}$ and $D M_{\mathrm{cc}}$ dropped suddenly and then recovered, although $D M_{\mathrm{cc}}$ was at a lower level than before plug ECRH turned on (red circles in Fig. 2). The sudden drops in $n \ell_{\mathrm{cc}}$ and $D M_{\mathrm{cc}}$ are explained by the formation of a non-axisymmetric potential in the central cell by plug ECRH [3].

Such sudden drops in $n \ell_{\mathrm{cc}}$ and $D M_{\mathrm{cc}}$, however, are also often observed when ECRH at the central cell (central ECRH) turns on in the GAMMA experiments. Central ECRH increases $D M_{\mathrm{cc}}$, so these sudden drops can be a result of a different mechanism from that of plug ECRH. Before central ECRH turns on, $D M_{\mathrm{cc}}$ is maintained at as high a level as possible. That is, a system with $\beta_{\perp \mathrm{c}}(0)$ and $\beta_{\perp \mathrm{A}}(0)$ (the ratio of plasma pressure to magnetic pressure at the central cell and anchor cell, respectively) exists around the stability boundary shown in Fig. 1. In this state, central ECRH can lead the system to the unstable region by increasing $D M_{\mathrm{cc}}$, destabilizing the flute modes. The excited flute mode fluctuations cause large plasma radial transport, which appears as sudden drops in $n \ell_{\mathrm{cc}}$ and $D M_{\text {cc }}$.

author's e-mail: katanuma@prc.tsukuba.ac.jp

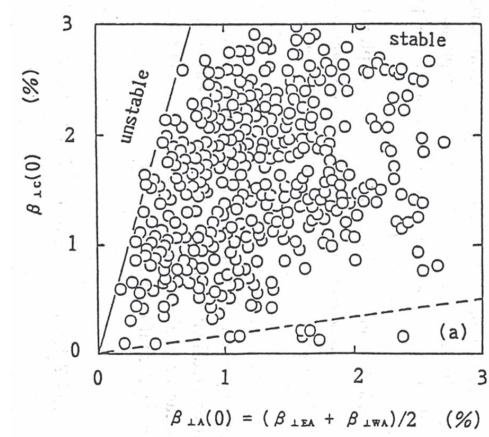

Fig. 1 Flute stability boundary of GAMMA10 plasma. Central cell on-axis beta value $\beta_{\mathrm{c}}(0)$ is shown versus anchor cell beta $\beta_{\mathrm{A}}(0)$, which is an arithmetical average of the east and west anchor betas. The symbol $\perp$ represents a component perpendicular to the magnetic field. The dashed line is the predicted boundary for an isotropic plasma in the central cell $\left(\beta_{\perp \mathrm{c}}=0.17 \beta_{\perp \mathrm{A}}\right)$; the solid line is that for an anisotropic plasma with an axially non-uniform pressure profile $\left(\beta_{\perp c}=4.0 \beta_{\perp \mathrm{A}}\right)$. The figure and caption also appear as Fig. 2 (a) in Ref. [1].

The mechanism of the $n \ell_{\mathrm{cc}}$ and $D M_{\mathrm{cc}}$ drops mentioned above must be verified. The purpose of this study is to clarify what kind of fluctuation appears and how much radial transport is caused by flute instabilities if the system becomes unstable with increasing $D M_{\mathrm{cc}}$.

\section{Interchange Mode}

In this section we briefly review the stability criterion for flute modes. The famous stability criterion for an axisymmetric system to the flute modes is given by [4]

$$
\int \frac{\mathrm{d} \ell}{\operatorname{Rr} B^{2}}>0 \text {. }
$$





Fig. 2 Time evolution of (a) east anchor line density, (b) central cell line density, and (c) central cell diamagnetism. The figure and caption appear as Fig. 6 in Ref. [2].

$R$ is the magnetic field line curvature, $r$ is the radius of a magnetic field line, $B$ is the magnitude of the magnetic field, and integration is done along a magnetic field line. The long thin approximation was not used to derive Eq. (1), so the criterion can be applied to a very fat mirror and a divertor magnetic mirror. However, Eq. (1) assumes isotropic plasma pressure.

On the other hand, the stability criterion of flute modes for a non-axisymmetric tandem mirror is given by [5]

$$
\int \frac{\left(\hat{p}_{\perp}+\hat{p}_{\|}\right) \kappa_{\psi}}{B} \mathrm{~d} \ell>0
$$

The plasma pressures $p_{\perp, \|}(\psi, B)$ are represented by separation of variables $p_{\perp, \|}(\psi, B)=\hat{p}_{\perp, \|}(B) v(\psi)$, where $p_{\perp, \|}$ are the components perpendicular and parallel to the magnetic field line, respectively; $\kappa_{\psi}$ is the normal curvature of a magnetic field line, i.e. $\hat{e}_{\|} \cdot \nabla \hat{e}_{\|}=\kappa=\kappa_{\psi} \nabla \psi+\kappa_{\theta} \nabla \theta$, where $\hat{e}_{\|}=\boldsymbol{B} /|B|$ and $\boldsymbol{B}=\nabla \psi \times \nabla \theta$. Although criterion (2) was obtained under the long thin approximation, it is applicable to a non-axisymmetric mirror with anisotropic pressure.

Equation (2) was used to determine the flute stability boundary of the GAMMA10 tandem mirror. It reduces to Eq. (1) for an axisymmetric mirror with isotropic pressure for the following reason. The covariant component $\kappa_{\psi}$ is expressed as $\kappa_{\psi}=\hat{e}_{\|} \cdot \nabla \hat{e}_{\|} \times \nabla \theta /(\nabla \psi \times \nabla \theta)$, so $\kappa_{\psi}=1 /(\operatorname{Rr} B)$ in an axisymmetric magnetic field. Equation (2), therefore, becomes Eq. (1) because an isotropic pressure is constant along the magnetic field lines.

Now we define the specific volume of a magnetic field line as $U \equiv \int(1 / B) \mathrm{d} \ell$. It is shown that $\partial U / \partial \psi=$ $-2 \int\left(\kappa_{\psi} / B\right) \mathrm{d} \ell$ in a vacuum magnetic field. Therefore, the stability criterion Eq. (1) is rewritten as $\partial U / \partial \psi<0$ if the definition of $U$ is changed to

$$
U \equiv \int \frac{\left(\hat{p}_{\perp}+\hat{p}_{\|}\right) \mathrm{d} \ell}{B}
$$

The stability criterion $\partial U / \partial \psi<0$ includes the anisotropic pressure, just like Eq. (2). GAMMA10 is an effectively axisymmetrized tandem mirror in the sense that $U$ defined in Eq. (3) is axisymmetric. Thus, the basic equations, which were obtained assuming an axisymmetric system, can be applied to GAMMA10 by using the definition of $U$ in Eq. (3).

\section{Basic Equations}

To calculate the flute mode fluctuations, the other modes (compressible Alfvén modes, shear Alfvén modes, and acoustic modes) are unnecessary, because those modes are more stable than flute modes; the Alfvén modes become unstable when $\beta \gtrsim 1$, where $\beta$ is the ratio of the plasma pressure to the magnetic pressure. It is, therefore, convenient to exclude the Alfvén and acoustic modes from the basic equations. The method for removing them from the magnetohydrodynamic (MHD) equations is given elsewhere $[6,7]$. The resulting equations are written

$$
\begin{aligned}
& \frac{\partial \hat{w}}{\partial t}+\llbracket \Phi, \hat{w} \rrbracket-\llbracket \hat{\rho},\left\langle\frac{v_{\alpha}^{2}}{2}\right\rangle \rrbracket+\frac{1}{U^{\gamma}} \frac{\partial U}{\partial \psi} \frac{\partial\left(\hat{\rho}_{0} \tilde{T}+\hat{T}_{0} \tilde{\rho}\right)}{\partial \varphi}=U Q_{w}^{*} \\
& +\frac{3 X_{M}}{20}\left(\frac{T_{\mathrm{i}}+T_{\mathrm{e}}}{2 T_{\mathrm{i}}}\right)\left\{\frac{\partial}{\partial \psi}\left(\hat{\rho}\left\langle r^{2}\right\rangle \frac{\partial}{\partial \psi}\left(\frac{1}{U^{2 / 3} \sqrt{\hat{T}_{0}}} \frac{a^{2}}{\left\langle r^{2}\right\rangle} \hat{w}\right)\right)\right. \\
& \left.+\frac{a^{2}}{\left\langle r^{2}\right\rangle}\left\langle\frac{1}{r^{2} B^{2}}+\lambda^{2} B^{2}\right\rangle \frac{\hat{\rho}}{U^{2 / 3} \sqrt{\hat{T}_{0}}} \frac{\partial^{2} \hat{w}}{\partial \varphi^{2}}\right\},
\end{aligned}
$$

Here $a^{2}=\left\langle r^{4}\right\rangle /\left\langle r^{2}\right\rangle$ and $\hat{w}$ has the form

$\hat{w}=\frac{\partial}{\partial \psi}\left(\hat{\rho}\left\langle r^{2}\right\rangle \frac{\partial \Phi}{\partial \psi}\right)+\frac{\partial}{\partial \varphi}\left(\hat{\rho}\left\langle\frac{1}{r^{2} B^{2}}+\lambda^{2} B^{2}\right\rangle \frac{\partial \Phi}{\partial \varphi}\right)$,

where $w$ is related to the specific volume-averaged vorticity $\nabla \times\left(\rho c \boldsymbol{B} \times \nabla \phi / B^{2}\right)$ of the plasma $\boldsymbol{E} \times \boldsymbol{B}$ drift flux. $v_{\alpha}^{2}$ is the square of the plasma fluid velocity. The Poisson bracket is defined as

$$
\llbracket \Phi, \hat{w} \rrbracket \equiv \frac{\partial \Phi}{\partial \psi} \frac{\partial \hat{w}}{\partial \varphi}-\frac{\partial \Phi}{\partial \varphi} \frac{\partial \hat{w}}{\partial \psi}
$$

where $\Phi \equiv c \phi$ with $c$ as light speed and $\phi$ as electrostatic potential. The second term on the right-hand side of Eq. (4) consists of the viscosity of fluid velocity and the effects by which the vorticity propagates along a magnetic null.

The transport equations of particles and heat are given as follows on assuming that the adiabatic index $\gamma=5 / 3$,

$$
\frac{\partial \hat{\rho}_{0}}{\partial t}-\frac{\partial}{\partial \psi}\left(\bar{\rho} \frac{\partial \Phi}{\partial \varphi}\right)=4 \pi d_{M} \frac{\partial}{\partial \psi}\left[\frac{\hat{\rho}_{0}\left\langle r^{2}\right\rangle U}{\hat{T}_{0}^{3 / 2}} \frac{\partial}{\partial \psi}\left(\frac{\hat{\rho}_{0} \hat{T}_{0}}{U^{5 / 3}}\right)\right]+\bar{Q}_{\rho}^{*} U
$$




$$
\begin{aligned}
& \frac{\partial \tilde{\rho}}{\partial t}+\llbracket \Phi, \tilde{\rho} \rrbracket+\frac{\partial}{\partial \psi}\left(\overline{\tilde{\rho} \frac{\partial \Phi}{\partial \varphi}}\right)-\frac{\partial \Phi}{\partial \varphi} \frac{\partial \hat{\rho}_{0}}{\partial \psi} \\
& =4 \pi d_{M} \frac{\partial}{\partial \psi}\left[\frac{\hat{\rho}_{0}\left\langle r^{2}\right\rangle U}{\hat{T}_{0}^{3 / 2}} \frac{\partial}{\partial \psi}\left(\frac{\tilde{\rho} \hat{T}_{0}}{U^{5 / 3}}\right)\right] \\
& +4 \pi d_{M}\left[\frac{\hat{\rho}_{0}}{\hat{T}_{0}^{1 / 2} U^{2 / 3}}\left\langle\frac{1}{r^{2} B^{2}}\right\rangle \frac{\partial^{2} \tilde{\rho}}{\partial \varphi^{2}}\right]+\tilde{Q}_{\rho}^{*} U, \\
& \frac{\partial \hat{T}_{0}}{\partial t}-\frac{\partial}{\partial \psi}\left(\overline{\tilde{T} \frac{\partial \Phi}{\partial \varphi}}\right)=X_{M} \frac{2 U^{2 / 3}}{3 \hat{\rho}_{0}} \frac{\partial}{\partial \psi}\left(\frac{\hat{\rho}_{0}^{2}\left\langle r^{2}\right\rangle}{U} \frac{\partial}{\partial \psi}\left(\frac{\sqrt{\hat{T}_{0}}}{U^{1 / 3}}\right)\right) \\
& +\frac{U^{5 / 3}}{\hat{\rho}} \bar{Q}_{T}^{*}+\frac{8 \pi d_{M}}{3 \sqrt{\hat{T}_{0}}} \frac{\partial}{\partial \psi}\left(\left\langle r^{2}\right\rangle U \frac{\partial}{\partial \psi}\left(\frac{\hat{\rho}_{0} \hat{T}_{0}}{U^{5 / 3}}\right)\right), \\
& \frac{\partial \tilde{T}}{\partial t}+\llbracket \Phi, \tilde{T} \rrbracket+\frac{\partial}{\partial \psi}\left(\overline{\tilde{T} \frac{\partial \Phi}{\partial \varphi}}\right)-\frac{\partial \Phi}{\partial \varphi} \frac{\partial \hat{T}_{0}}{\partial \psi} \\
& =X_{M} \frac{U^{2 / 3}}{3 \hat{\rho}_{0}} \frac{\partial}{\partial \psi}\left(\frac{\hat{\rho}_{0}^{2}\left\langle r^{2}\right\rangle}{U} \frac{\partial}{\partial \psi}\left(\frac{\tilde{T}}{U^{1 / 3} \sqrt{\hat{T}_{0}}}\right)\right) \\
& +\frac{X_{M} \hat{\rho}_{0}}{3 U^{2 / 3} \sqrt{\hat{T}_{0}}}\left\langle\frac{1}{r^{2} B^{2}}\right\rangle \frac{\partial^{2} \tilde{T}}{\partial \varphi^{2}}+\frac{U^{5 / 3}}{\hat{\rho}} \tilde{Q}_{T}^{*} .
\end{aligned}
$$

The mass density is defined as $\rho \equiv M_{\mathrm{i}} n_{\mathrm{i}}+M_{\mathrm{e}} n_{\mathrm{e}}=\langle\rho\rangle$ $\equiv \hat{\rho} / U$. A slow time-variable equilibrium component $\hat{\rho}_{0}\left(\epsilon^{3} t, \psi\right)$ and fast variable fluctuating components $\tilde{\rho}(\epsilon t, \psi, \varphi)$ are defined, where the equilibrium quantity is represented by a hat with subscript $\hat{0}_{0}$, and fluctuating quantities are represented by a tilde $\sim$, e.g., $\hat{\rho}=\hat{\rho}_{0}+\tilde{\rho}$. The quantity $\hat{T}$ is related to the temperature defined by $\hat{T} \equiv p U^{\gamma} / \hat{\rho}=\left(T_{\mathrm{i}}+T_{\mathrm{e}}\right) U^{\gamma-1} / M_{\mathrm{i}}$. The symbol $\bar{A}$ indicates the average of $A$ over $\varphi, \hat{A}$ denotes $A$ integrated along a magnetic field line, and $\langle A\rangle$ indicates the integration of $A$ along a magnetic field line,

$$
\bar{A} \equiv \frac{1}{2 \pi} \int_{0}^{2 \pi} A \mathrm{~d} \varphi, \hat{A} \equiv \int \frac{A \mathrm{~d} \zeta}{J(\psi, \varphi, \zeta)},\langle A\rangle \equiv \frac{\hat{A}}{U} .
$$

The symbol $J(\psi, \varphi, \zeta)$ is the Jacobian $J(\psi, \varphi, \zeta) \equiv \nabla \psi \times$ $\nabla \varphi \cdot \nabla \zeta$. The magnetic field line curvatures are included in the coefficients $U,\left\langle r^{2}\right\rangle$ and $\left\langle\frac{1}{r^{2} B^{2}}\right\rangle$ in Eqs. (4)-(10), and $U$ is defined as

$$
U \equiv \int \frac{\left(\hat{p}_{\perp}+\hat{p}_{\|}\right) \mathrm{d} \zeta}{J(\psi, \varphi, \zeta)} .
$$

The coordinates $(\psi, \varphi, \zeta)$ adopted here are the flux coordinates, where the magnetic field is given as $\boldsymbol{B}=\nabla \psi \times \nabla \varphi$. $2 \pi \psi$ gives the magnetic flux inside a surface of $\psi=$ const, and $\varphi$ corresponds to an angular coordinate. The remaining coordinate $\zeta$ is taken as the $z$-axis or along magnetic field lines $[8,9]$.

The classical viscosity is included in Eqs. (4)-(10) by

$$
\begin{aligned}
& X_{M}=\chi_{\perp} \frac{B^{2}}{\rho}\left(\frac{4 T_{\mathrm{i}}}{T_{\mathrm{i}}+T_{\mathrm{e}}}\right)\left(\frac{p}{\rho}\right)^{1 / 2}, \\
& d_{M} \equiv \frac{m_{\mathrm{e}}^{1 / 2}\left(T_{\mathrm{i}}+T_{\mathrm{e}}\right) T_{\mathrm{i}}^{1 / 2}}{\sqrt{2} m_{\mathrm{i}}^{1 / 2} T_{\mathrm{e}}^{3 / 2}}\left(\frac{B^{2}}{4 \pi \rho}\left(\frac{p}{\rho}\right)^{1 / 2} \chi_{\perp}\right), \\
& \chi_{\perp}=\frac{T_{\mathrm{i}}}{m_{\mathrm{i}} \omega_{\mathrm{ci}}^{2} \tau_{\mathrm{i}}}, \quad \epsilon^{3} \equiv \frac{\chi_{\perp}}{b c_{s}}\left(\frac{2 T_{\mathrm{i}}}{T_{\mathrm{i}}+T_{\mathrm{e}}}\right) .
\end{aligned}
$$

$\tau_{\mathrm{i}}$ is the classical ion-ion Coulomb collision time [10], $c_{\mathrm{S}}$ is the ion sound speed $c_{\mathrm{s}} \equiv \sqrt{\gamma p / \rho}$, and $b$ has a dimension of distance defined by $b \equiv \sqrt{\psi_{\mathrm{b}} / B_{M}}$, where the subscript ${ }_{M}$ represents some axial position on the axis and $\psi_{\mathrm{b}}$ is the coordinate at the limiter (wall). The quantities related to classical transport, $X_{M}$ and $d_{M}$ in Eq. (13), are non-dimensional and are constant along a magnetic field line. The mass density $\rho$ and other plasma quantities $T_{\mathrm{e}}$ and $T_{\mathrm{i}}$ are assumed to be constant along a magnetic field line throughout this paper. The parameter $\epsilon$ defined in Eq. (14) is a small expansion parameter, where we assume $\epsilon^{2}=10^{-2}$.

The basic equations in this section contain the flute modes (similar to the Rayleigh-Taylor instabilities) and the modes associated with the presence of nonuniform plasma flows (similar to the Kelvin-Helmholtz instabilities) as well as the electrostatically incompressible stable plasma flows. Thus this closed set of equations describes nonlinear low-frequency MHD plasma convection and the resulting transport processes in weakly dissipative plasmas in axisymmetric shearless systems.

The local dispersion obtained by these reduced equations is

$$
\begin{aligned}
& \left(\omega-m \frac{\partial \Phi_{0}}{\partial \psi}\right)^{2}\left(m^{2} \hat{\rho}_{0}\left\langle\frac{1}{r^{2} B^{2}}+\lambda^{2} B^{2}\right\rangle\right) \\
& +\left(\omega-m \frac{\partial \Phi_{0}}{\partial \psi}\right)\left(m \frac{\partial \hat{\rho}_{0}}{\partial \psi}\left(\frac{\partial}{\partial \psi}\left(\left\langle r^{2}\right\rangle \frac{\partial \Phi_{0}}{\partial \psi}\right)\right)-m \frac{\partial \hat{w}_{0}}{\partial \psi}\right) \\
& -\left(\frac{m^{2}}{2} \frac{\partial \overline{\left\langle v_{\alpha}^{2}\right\rangle}}{\partial \psi} \frac{\partial \hat{\rho}_{0}}{\partial \psi}+\frac{m^{2}}{U^{\gamma}} \frac{\partial U}{\partial \psi} \frac{\partial\left(\hat{\rho}_{0} \hat{T}_{0}\right)}{\partial \psi}\right)=0
\end{aligned}
$$

If the coefficient of $\left(\omega-m \frac{\partial \Phi_{0}}{\partial \psi}\right)$ is small, the condition for positive eigen-value $\omega^{2}$ in Eq. (16) is

$$
\left(\frac{m^{2}}{2} \frac{\partial \overline{\left\langle v_{\alpha}^{2}\right\rangle}}{\partial \psi} \frac{\partial \hat{\rho}_{0}}{\partial \psi}+\frac{m^{2}}{U^{\gamma}} \frac{\partial U}{\partial \psi} \frac{\partial\left(\hat{\rho}_{0} \hat{T}_{0}\right)}{\partial \psi}\right)<0 .
$$

When the condition is not satisfied, instability resulting from velocity shear and density shear (Kelvin-Helmholtz instability) and flute instability (Rayleigh-Taylor instability) occurs.

\section{Numerical Results}

We apply the basic equations (4)-(10) to GAMMA10 to investigate the influence of flute mode fluctuations on the saturation of $n \ell_{\mathrm{cc}}$ and $D M_{\mathrm{cc}}$. The axial pressures adopted here are plotted in Fig. 3, where

$\hat{p}_{\perp}(B)+\hat{p}_{\|}(B)= \begin{cases}\max \left(p_{A} \frac{B_{\mathrm{m}}^{2}-B^{2}}{B_{\mathrm{m}}^{2}-B_{\mathrm{c}}^{2}}, p_{\mathrm{c}}\right) & \text { in } B \leq B_{\mathrm{m}} \text { of Anchor } \\ p_{\mathrm{c}} & \text { in other region }\end{cases}$

$B_{\mathrm{c}}$ is the magnetic field at the anchor midplane on the axis and $B_{\mathrm{m}}=1.7 \times B_{\mathrm{c}}$, and $B$ is the magnitude of the magnetic field line in the $x-z$ plane.

Figure 3 plots the axial profiles of $\hat{p}_{\perp}+\hat{p}_{\|}$at various radii, which are used to calculate the specific volume of a 


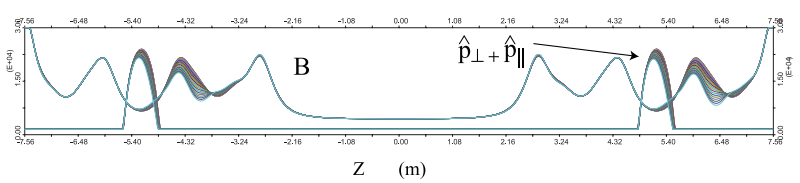

Fig. 3 Axial magnetic field and axial pressure in GAMMA10. Different colors correspond to different radial magnetic field lines. $p_{\mathrm{A}}=4$ and $p_{\mathrm{c}}=1$.

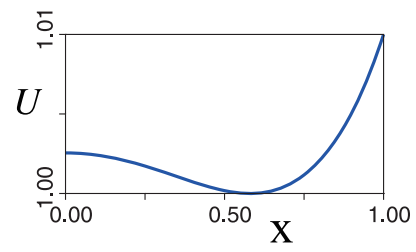

Fig. 4 Radial profile of the specific volume defined in Eq. (12).

magnetic field line in Eq. (12). The resulting specific volume is plotted in Fig. 4 , where $\hat{p}_{\perp}+\hat{p}_{\|}=p_{\mathrm{A}}$ at the anchor midplane on the axis, and $\hat{p}_{\perp}+\hat{p}_{\|}=p_{\mathrm{c}}$ at the central cell midplane. The central cell pressure $p_{\mathrm{c}}$ is determined by the relationship of $p_{\mathrm{c}}=\hat{\rho}_{0}(0) \hat{T}_{0}(0)$, where $\hat{\rho}_{0}(0)$ and $\hat{T}_{0}(0)$ are obtained in the simulation.

The initial condition is that $\hat{\rho}_{0}(x)=1 \times u(x), \hat{T}_{0}(x)=$ $0.25 \times \exp \left\{-x^{2}\right\} \times u(x)^{\gamma-1}, \hat{w}_{0}(x)=+1.0$, and very small perturbations are added to $\tilde{T}(x)$ initially, The boundary condition is that $\hat{\rho}\left(x_{\text {wall }}\right)=1 \times u\left(x_{\text {wall }}\right), \hat{T}\left(x_{\text {wall }}\right)=0.25 \times$ $\exp \left\{-x_{\text {wall }}^{2}\right\} \times u\left(x_{\text {wall }}\right)^{\gamma-1}$.

We simulated three cases of external sources $Q_{t 0}(x)$ and $Q_{d 0}(x)$, where $x=r / r_{\text {wall }}, r_{\text {wall }}=18 \mathrm{~cm}$ at the central cell midplane, and $x_{\text {wall }}=1$. The first case is a weak external source; $Q_{t 0}(x)=0.25 \times \exp \left\{-2 x^{2}\right\}, Q_{d 0}(x)=$ $0.125 \times \exp \left\{-x^{2}\right\}$. The second case is a medium-strength external source; $Q_{t 0}(x)=0.5 \times \exp \left\{-2 x^{2}\right\}, Q_{d 0}(x)=0.25 \times$ $\exp \left\{-x^{2}\right\}$. The third and fourth cases are a strong external source; $Q_{t 0}(x)=\exp \left\{-2 x^{2}\right\}, Q_{d 0}(x)=0.5 \times \exp \left\{-x^{2}\right\}$. Henceforth, the normalized time $\tau$ defined as $\tau=\frac{\epsilon c_{S M}}{b} t$ is used.

Figures 5, 6, 7, and 8 show the time evolution of density and temperature at various radial positions. $\hat{T}_{0}$ and $\hat{\rho}_{0}$ are the zeroth-order temperature and density, respectively, at $x=0,1 / 3,1 / 2$, and $2 / 3$, where zeroth order quantities are measured at $\varphi=0$. The Fourier amplitudes of the perturbed quantities $\tilde{\rho}$ and $\tilde{T}$, which are measured at $x=1 / 2$, grow in time and enter the nonlinear phase quickly in every figure.

For the weak external source, as shown in Fig. 5, the temperature $\hat{T}_{0}$ and density $\hat{\rho}_{0}$ are supplied by the external source and lost by classical diffusion; they enter a steady state in which both are balanced. On the other hand, as seen in Fig. 6 for the medium-strength external source, a steady state is realized in which the external sources and radial transport due to flute mode fluctuations are balanced. The perturbations of temperature $\tilde{T}$ and density $\tilde{\rho}$ grow in time until $\tau \simeq 270$ and correspond to the build up of the
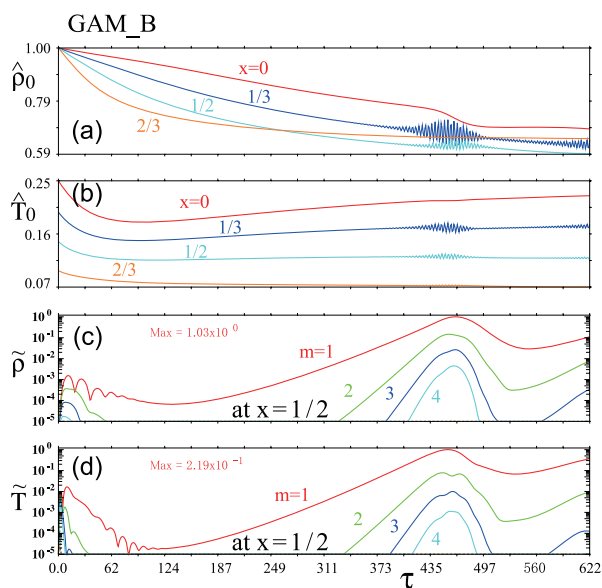

Fig. 5 Case of weak external source. $\hat{\rho}_{0}$ and $\hat{T}_{0}$ were obtained at $x=0,1 / 3,1 / 2$, and $2 / 3$ in (a), (b), and $m=1 \sim 4$ Fourier amplitudes of $\tilde{\rho}$ and $\tilde{T}$ were obtained at $x=1 / 2$ in (c), (d).
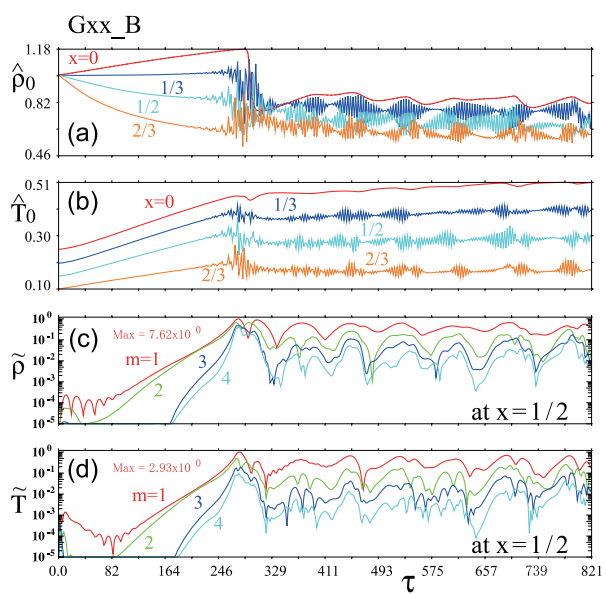

Fig. 6 Case of medium-strength external source. $\hat{\rho}_{0}$ and $\hat{T}_{0}$ were obtained at $x=0,1 / 3,1 / 2$, and $2 / 3$ in (a), (b), and $m=$ $1 \sim 4$ Fourier amplitudes of $\tilde{\rho}$ and $\tilde{T}$ were obtained at $x=1 / 2$ in (c), (d).

zeroth-order temperature $\hat{T}_{0}$ and density $\hat{\rho}_{0}$; they then enter a steady state. This steady state in which the external source input is balanced with the radial loss by flute mode fluctuations is first found in this simulation in the GAMMA10 magnetic configuration.

For the strong external source, as shown in Fig. 7, the situation differs from that of a medium-strength external source. When the amplitudes of the perturbation quantities are maximum in time, $\tau \simeq 100$, a very large radial transport appears, which leads to the break down of plasma in the simulation.

The result of a simulation with the same strong external source of Fig. 7 but different boundary conditions is shown in Fig. 8. The boundary condition differs from that of Fig. 7 in that $\hat{T}_{\text {wall }}=0.5 \times \exp \left\{-2 x_{\text {wall }}^{2}\right\} \times u_{\text {wall }}^{\gamma-1}$ is used, which is larger than that of Fig. 7. The maximum of $m=1$ Fourier amplitude of $\tilde{\rho}$ in Fig. 8 (c) is smaller than that of Fig. 7 (c) because the density and temperature 

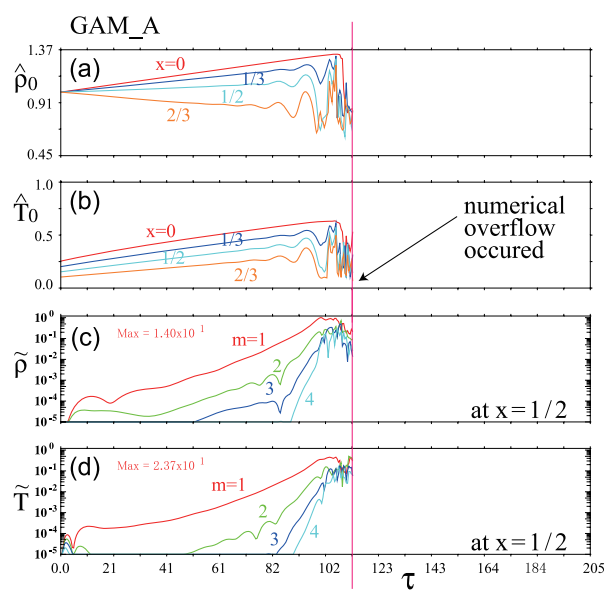

Fig. 7 Case of strong external source. $\hat{\rho}_{0}$ and $\hat{T}_{0}$ were obtained at $x=0,1 / 3,1 / 2$, and $2 / 3$ in (a), (b), and $m=1 \sim 4$ Fourier amplitudes of $\tilde{\rho}$ and $\tilde{T}$ were obtained at $x=1 / 2$ in (c), (d).
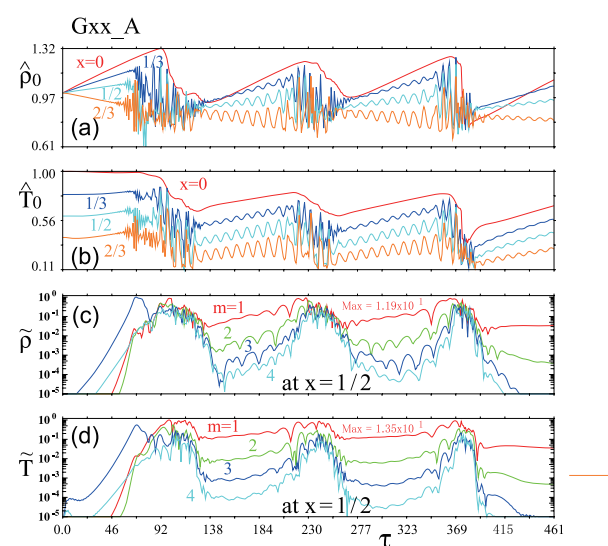

Fig. 8 Case of different boundary conditions in addition to a strong external source. $\hat{\rho}_{0}$ and $\hat{T}_{0}$ were obtained at $x=0$, $1 / 3,1 / 2$, and $2 / 3$ in (a), (b), and $m=1 \sim 4$ Fourier amplitudes of $\tilde{\rho}$ and $\tilde{T}$ were obtained at $x=1 / 2$ in (c), (d).

at the boundary are set higher than those in Fig. 7. The boundary condition of higher $\hat{T}_{\text {wall }}$ in Fig. 8 than in Fig. 7 weakens the gradient of $\hat{T}$ near the boundary, which results from classical diffusion. Thus, the growth rate of flute instabilities is lower than that in Fig. 7. However, the effects of flute mode fluctuations on the plasma in Fig. 8 are clearer, that is, the increase in the zeroth-order temperature $\hat{T}_{0}$ and density $\hat{\rho}_{0}$ makes the flute modes unstable. enhancing radial transport; then the zeroth-order temperature $\hat{T}_{0}$ and density $\hat{\rho}_{0}$ decrease. The flute mode fluctuations have high amplitudes during the decreases in $\hat{T}_{0}$ and $\hat{\rho}$, as seen in Fig. 8. That is, $\hat{T}_{0}$ and $\hat{\rho}_{0}$ continue to decrease rapidly in the phase during which the amplitudes of the flute modes fluctuations are high.

Figure 9 shows the radial profiles of $U, \hat{T}_{0}$, and $\hat{\rho}_{0}$ at time $\tau=140,180,220$, and 260, which correspond to Fig. 8. At $\tau=140$, the magnitudes of the zeroth-order temperature and density in Fig. 8 are low; they grow in time because of the external source until $\tau=230$.

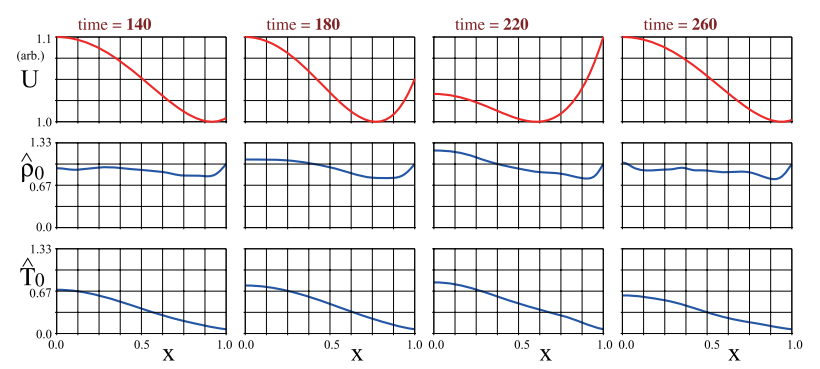

Fig. 9 Radial profiles of zeroth order quantities $U, \hat{\rho}_{0}$, and $\hat{T}_{0}$ in the case of different boundary conditions in addition to a strong external source. $U$ is plotted in arbitrary units.

The specific volume $U$ in Fig. 9 shows that the flute modes become unstable in the peripheral region at $\tau=180$, and the unstable region expands until $\tau=220$, when the perturbed amplitude is maximum and flute mode fluctuations lead to large radial transport. As the zeroth-order temperature and density are low because of the large radial transport, the specific volume has a radial profile stable to flute modes at $\tau=260$. These processes repeated in Fig. 8 .

\section{Summary}

A reduced MHD simulation was performed to investigate the influence of flute instability on radial transport in GAMMA10. A steady state occured in which an external source is balanced with the radial transport caused by flute mode fluctuations. A strong external source enhances the strong flute instability, causing large radial transport, and a steady state with sawtooth-like oscillation of the zeroth order density and temperature is realized. Some sudden drops in $D M_{\mathrm{cc}}$ observed in GAMMA10 may be explained by flute instability.

[1] M. Inutake et al., Nuclear Fusion Supplement 2, 651 (1993), (Proc. 4th Int. Conf. on Plasma Physics and Controlled Nuclear Fusion Research, IAEA, Würzburg, 30 Sep.-7 Oct., 1992).

[2] K. Yatsu et al., Nucl. Fusion 41, 613(2001).

[3] I. Katanuma, K. Yashiro, T. Imai and V.P. Pastukhov, Proc. 18th Int. Toki Conf. Development of Physics and Technology of Stellarators/Heliotrons "en route to DEMO", Ceratopia Toki, Gifu, Japan, December 9-12, 2008, P1-16, pp.200-203.

[4] for example, M.N. Rosenbluth and C.L. Longmire, Annals of Physics 1, 120 (1957).

[5] for example, I. Katanuma, Y. Tatematsu, K. Ishii, T. Tamano and K. Yatsu, J. Phys. Soc. Jpn. 69, 3244 (2000).

[6] P.V. Pastukhov, Plasma Phys. Rep. 31, 577 (2005).

[7] I. Katanuma, P.V. Pastukhov et al., J. Plasma Fusion Res. 84, 279 (2008).

[8] I. Katanuma, Y. Sasagawa, Y. Tatematsu et al., Nucl. Fusion 46, 608 (2006).

[9] Y. Sasagawa, I. Katanuma, Y. Mizoguchi et al., Phys. Plasmas 13, 122506 (2006).

[10] S.I. Braginskii, Reviews of Plasma Physics, ed. A.M.A. Leontovich (Consultants Bureau, New York, 1965) Vol. 1. 